\title{
The Use of Spectral-Domain Optical Coherence Tomography to Detect Glaucoma Progression
}

\author{
Ricardo Y. Abe ${ }^{1,2}$, Carolina P.B. Gracitelli ${ }^{1,3}$ and Felipe A. Medeiros ${ }^{*}, 1$ \\ ${ }^{1}$ Hamilton Glaucoma Center and Department of Ophthalmology, University of California, San Diego, La Jolla, \\ California, USA \\ ${ }^{2}$ Department of Ophthalmology, University of Campinas, Campinas, Brazil \\ ${ }^{3}$ Department of Ophthalmology, Federal University of São Paulo, São Paulo, Brazil
}

\begin{abstract}
Detection of progression and measurement of rates of change is at the core of glaucoma management, and the use of Spectral Domain Optical Coherence Tomography (SD-OCT) has significantly improved our ability to evaluate change in the disease. In this review, we critically assess the existing literature on the use of SD-OCT for detecting glaucoma progression and estimating rates of change. We discuss aspects related to the reproducibility of measurements, their accuracy to detect longitudinal change over time, and the effect of aging on the ability to detect progression. In addition, we discuss recent studies evaluating the use of combined structure and function approaches to improve detection of glaucoma progression.
\end{abstract}

Keywords: Glaucoma, progression, OCT, spectral domain optical coherence tomography.

\section{INTRODUCTION}

According to the World Health Organization, glaucoma is the second leading cause of preventable blindness globally [1]. The disease is characterized by a degenerative optic neuropathy associated with progressive loss of retinal ganglion cell (RGC) axons, leading to losses in visual function [2]. Due to the irreversible nature of glaucomatous damage, early diagnosis and adequate treatment are important for decreasing the risk of visual impairment or blindness from the disease [2]. Proper monitoring and detection of progression is paramount, and assumes a central role in the management of patients diagnosed with glaucoma or suspected of having the disease [3].

Standard automated perimetry (SAP) has traditionally been used as the gold standard for evaluation of progressive damage in glaucoma [4]. However, several large prospective clinical studies have shown that many patients can present progressive structural optic nerve or retinal nerve fiber layer (RNFL) damage without detectable losses on SAP [5-8]. In many patients, progressive structural damage frequently precedes functional deterioration, giving clinicians an opportunity to start or intensify treatment before the disease causes significant functional deterioration [9]. However, detecting glaucoma progression can be challenging, and there is currently no consensus on how to define and evaluate progressive change in the disease [10].

Although optic disc photographs have been used to monitor structural progression, they are limited by the need

*Address correspondence to this author at the Hamilton Glaucoma Center University of California, San Diego, 9500 Gilman Drive La Jolla, CA 92093-0946, USA; Tel: 858-822-4592; Fax: 858-822-0615;

E-mail: fmedeiros@glaucoma.ucsd.edu for subjective assessment [11]. In fact, previous reports have described suboptimal reproducibility for the assessment of optic nerve deterioration when different or even the same observers evaluate change using optic disc photographs $[12,13]$. In addition, evaluation of progression using optic disc photographs does not allow an objective quantification of the rate of disease progression over time. Although most glaucoma patients will show some evidence of progression if followed long enough, the rate of deterioration can be highly variable among them [14]. While most patients progress relatively slowly, others have aggressive disease with fast deterioration, which can eventually result in blindness or substantial impairment unless appropriate interventions take place [15].

The use of imaging technologies has allowed objective and quantitative assessment of structural damage in glaucoma [3]. Optical coherence tomography (OCT) is an imaging technology that has been used for assessment of progression and rates of deterioration in the disease [16-19]. The OCT technology has undergone major advancements in the recent past, from timedomain to spectral-domain OCT, as well as the recent development of swept-source OCT and other variations of the technology [20]. These advancements have resulted in increased reproducibility and accuracy in the assessment of progressive damage, not only to RNFL, but also to other regions affected by glaucomatous damage, such as the optic nerve head $(\mathrm{ONH})$ and macula [21, 22]. In this review, we critically evaluate the available evidence with regard to the ability of different OCT parameters in detecting glaucomatous progression and evaluating rates of change in the disease.

\section{REPRODUCIBILITY}

In order to be able to assess progression, test measurements need to have adequate reproducibility [23-25]. 
If test measurements show poor reproducibility with too much test-retest variability, detection of true deterioration will be challenging due to the difficulty in separating the progression signal from the noise introduced by variability. Intra-class correlation coefficient (ICC), coefficient of variation $(\mathrm{CV})$ and test-retest variability (TRV) are some of the statistical methods that can be used to assess variability, reproducibility and repeatability of measurements [25, 26]. ICC is a statistic that summarizes the reproducibility of a measurement process based on a variance components analysis, expressing the variance attributed to real differences between subjects as a fraction of the total variation, which also includes sources of measurement variability [26]. In brief, a large ICC indicates good reproducibility, with only relatively small fluctuations among repeated measurements on the same subjects [27]. The maximum value of the ICC is 1 (or $100 \%$ ), whereas its minimum value is theoretically 0 . The $\mathrm{CV}$ is defined as the standard deviation divided by the mean. It is expressed as a percentage (\%), where higher values means greater dispersion in the variable, that is, greater variability [26]. A measurement with a COV $<10 \%$ is considered to have good reproducibility. Finally, the TRV can be calculated as two times the standard deviation of repeated measurements. A measurement with low TRV has, as the name implies, low variability and good reproducibility [26].

Pierro et al. evaluated RNFL thickness measurements using 7 different OCTs (spectral and time-domain), to assess inter and intraoperator reproducibility of 38 healthy subjects [24]. For interoperator reproducibility, average RNFL thickness measurements had ICCs ranging from 0.57 to 0.92 , with CV ranging from $1.65 \%$ to $3.35 \%$. For intraoperator reproducibility, ICCs ranged from 0.70 to 0.97 and COV from $1.16 \%$ to $2.18 \%$. The same author also studied 18 healthy subjects to analyze interoperator and intraoperator reproducibility of OCT measurements for average macular thickness and revealed ICCs ranging from 0.75 to 0.97 and $\mathrm{CV}$ from $0.43 \%$ to $2.75 \%$ [28]. In another investigation, Mwanza et al. examined 55 eyes of glaucoma patients evaluating intra-visit and inter-visit reproducibility of circumferential RNFL and ONH measurements [29]. ICCs ranged from 0.83 to 0.99 for intra-visit and 0.80 to 0.99 for inter-visit measurements. They estimated that inter-visit thinning of at least $4 \mu \mathrm{m}$ for RNFL measurements may be considered as suspicious change occurring as the result of glaucoma progression. Other authors have also conducted studies supporting high reproducibility of OCT measurements [30-33].

It is important to note that although many studies have indicated a high reproducibility of a variety of OCT measurements, these studies have in general only evaluated short-term reproducibility $[24,29,30]$. That is, patients underwent test-retest over relatively short periods of time. This was done in order to eliminate or decrease the risk that true disease progression could confound the assessment of reproducibility. As glaucoma is generally a relatively slowly progressive disease, it is unlikely that patients would present true deterioration if tested over short periods of time. However, in clinical practice, evaluation of progression occurs over long periods of time and it is also important to ascertain whether the long-term reproducibility is also adequate $[34,35]$. There is no guarantee that long-term reproducibility will be as high as short-term reproducibility. In fact, it is likely that intercurring factors and patient cooperation may cause an increase in test-retest variability over long periods of time. Unfortunately, evaluation of longterm reproducibility can be challenging, due to the possible confounding effects of concomitant disease progression. However, strategies have been proposed to overcome these confounding effects and previous studies have suggested that long-term reproducibility may actually be lower than the reproducibility reported in short-term studies [34].

\section{DETECTING PROGRESSION WITH OCT}

OCT was first described by Huang et al. in 1991 and it uses a low-coherence interferometry to produce a twodimensional image of optical scattering from internal tissue microstructures in a way that is analogous to ultrasonic pulse-echo imaging [36]. Initial versions of this technology employed what is called time-domain OCT (TD-OCT) (Stratus OCT, Carl Zeiss Meditec Inc, Dublin, CA) [37]. In TD-OCT, depth information of the retina is obtained after a longitudinal translation in time of a reference arm [37]. TDOCT is limited by a scan rate of only 400 axial scans per second generating a topographic map of the RNFL around the optic disc, with an axial resolution of 8 to $10 \mu \mathrm{m}$ and a transverse resolution of approximately $20 \mu \mathrm{m}$. In contrast, spectral domain OCT (SD-OCT) measures the interferometric signal detected as a function of optical frequencies, allowing for imaging speeds 50 times faster than TD-OCT, providing a greater number of images per unit area $[38,39]$. With SD-OCT, better resolution and faster scanning speed allow high-density raster scanning of retinal tissue while minimizing eye motion artifacts [40]. This device avoids moving the reference arm and uses a spectrometer on the detector arm to measure the difference in wavelength between the light from the fixed reference arm and that returning from the tissue [40]. In addition, Fourier analysis is used to analyze images according to the recorded wavelength of light [39]. It can acquire a high number of scans per second (27.000 - 40.000 A scans per second) and recent studies have demonstrated excellent intra-visit and inter-visit measurement reproducibility for SD-OCT, superior to TD-OCT [41-43].

Wollstein et al. conducted a retrospective study to evaluate glaucoma progression using a prototype version of TD-OCT technology [44]. Patients had an average of 4.7 years of follow up (55 eyes from 32 glaucoma patients and 9 eyes from 5 glaucoma suspects). Progression of OCT was defined as a repeatable average RNFL thinning of at least 20 $\mu \mathrm{m}$. This value was calculated based on twice the estimated reproducibility error of the device $(10 \mu \mathrm{m})$. They concluded that there was a greater likelihood of glaucomatous progression as measured by OCT compared with visual field assessment. However, this study was largely limited by the lack of any attempt to use an independent source to validate progression, making it difficult to ascertain whether the excess of cases detected as progression by the prototype OCT was due to true disease deterioration or just falsepositives.

Subsequent studies evaluated the ability of detecting glaucoma progression using commercially available OCT devices [16, 45]. Medeiros et al. evaluated 253 eyes of 
patients with glaucoma or suspected of disease with mean follow-up time of 4.1 years using Stratus TD-OCT (Carl Zeiss Meditec, Dublin, California, USA) measuring RNFL, $\mathrm{ONH}$ and macular thickness [16]. Glaucoma progression was determined by using SAP and masked optic disc stereophotographs. The results showed that mean rates of change in average RNFL thickness were significantly faster for eyes with progressive disease compared to nonprogressors. RNFL parameters also performed significantly better than $\mathrm{ONH}$ and macular thickness measurements in discriminating progressors from nonprogressors.

A major limitation of TD-OCT technology was that measurements of RNFL thickness were frequently obtained at different locations when the test was repeated in the same patients over time. This occurred because of poor ability of registering the images acquired over time from the same patient using anatomical landmarks. As the measurements were acquired over different locations, this frequently resulted in poor reproducibility and decreased ability to detect true change over time [46, 47]. The improved scan speed and resolution of SD-OCT has greatly improved the ability to register images of the same patient over time. That is, anatomical landmarks are now used very efficiently to ensure that the same location of the retina is imaged and that measurements are always obtained over the same location of interest $[48,49]$.

A previous study evaluated SD OCT reproducibility and assessed the agreement of RNFL measurements between SD-OCT and TD-OCT [41]. The study found a good reproducibility for $\mathrm{SD}-\mathrm{OCT}$, with $\mathrm{CV}$ s ranging from $1.5 \%$ to $6.6 \%$ and ICCs from 0.81 to 0.98 for average RNFL thickness. Average RNFL thickness measurements were highly correlated $\left(\mathrm{R}^{2}=0.92\right)$ between the two instruments. However, Bland-Altman plots revealed that the measurements were not interchangeable, a finding also confirmed in other studies [42, 43]. That is, one should not directly compare measurements obtained with previous versions of the OCT technology with those obtained by the different SD-OCT devices. A recent study has proposed conversion formulas that could be used for this purpose [50]. However the use of these formulas should be made with caution, as their impact has not been validated for assessment of individual progression.

Several different commercially available SD-OCT devices can provide an assessment of glaucoma progression $[17,45,51]$. There is no clear evidence supporting one device over the others in the ability to detect progressive damage in the disease. Although the methods used for calculation of several structural parameters are similar among the devices, each device provides specific parameters that are proposed to assist in detecting damage and diagnosing progression [52]. In addition, each device has its own unique normative database and there are large differences regarding the number and clinical characteristics of the eyes included in the databases [52]. Below, we review the current evidence with regard to the ability of detecting progression for some of the commercially available OCT devices that have been most studied at this point.

\section{SPECTRALIS SD-OCT}

The Spectralis SD-OCT (Heidelberg Engineering, Heidelberg, Germany) is able to obtain OCT scans with an impressive speed of 40,000 A-scans/second. The device has an axial resolution of $3.9 \mu \mathrm{m}$, with transverse resolution of $14 \mu \mathrm{m}$ and scan depth of $1.9 \mathrm{~mm}$. The light source used is a super luminescent diode centered at a wavelength of $870 \mathrm{~nm}$ [40]. The Spectralis utilizes a dual-beam scanning system consisting of a confocal scanning laser ophthalmoscopy (CSLO) reference beam to acquire reference scans for eye movement tracking and also a second beam to simultaneously acquire OCT images. The eye-tracking technology recognizes the presence of eye movement and discards scans with motion artifacts [40]. This system results in a very efficient method to ensure that the same areas of interest are scanned over time. In addition, Spectralis also oversamples specific points on the OCT scans and subsequently compares and combines them to reduce speckle (or random noise), enhancing the visualization of structures of interest. RNFL thickness measurements are obtained by averaging 16 consecutive circular B-scans of a $3.4 \mathrm{~mm}$ diameter peripapillary circle centered on the optic disc. The instrument provides a "RNFL Change Report" that includes individual baseline and follow-up scans for the overall and sectorial RNFL measurements and classifications. Fig. (1) shows an example of a glaucoma patient followed for approximate 2 years showing RNFL Change Report with worsening of the average, temporal superior and inferior RNFL thickness.

Wessel et al. investigated 62 eyes of 38 open angle glaucoma patients and 24 healthy controls for an average period of 3 years with annual visits for Spectralis SD-OCT, measuring circumferential RNFL thickness [19]. They divided the eyes into two groups (progressors and nonprogressors) according to masked comparative analysis of photographs. Using trend-based analysis, they revealed a rate of progression of $-2.12 \mu \mathrm{m}$ per year in patients with progressive optic disc changes, which was significantly faster when compared to glaucoma patients without progression $(-1.18 \mu \mathrm{m} /$ year, $\mathrm{P}=0.002)$ and with healthy controls $(-0.60 \mu \mathrm{m}, \mathrm{P}<0.001)$. The difference in RNFL loss between progressing and non-progressing glaucoma eyes was maximal in the inferior optic disc sectors. Since RNFL thickness also decreased in patients without progression, the authors observed that a longer observation time of these patients might be able to reveal whether the Spectralis is actually detecting progression that is not being detected by optic disc photographs.

Miki et al. evaluated 554 eyes from 294 glaucoma suspects for a mean period of 2.2 years using Spectralis SDOCT and compared rates of RNFL loss in the group who developed visual field defects against the other who did not developed changes in visual field [53]. The estimated mean rate of global RNFL loss was significantly faster in the eyes that developed visual field defects compared with eyes that did not develop visual field defect $(-2.02 \mu \mathrm{m} /$ year versus $-0.82 \mu \mathrm{m} /$ year; $P<0.001)$. Using joint longitudinal survival model, they showed that each $1 \mu \mathrm{m} /$ year faster rate of global RNFL loss corresponded to a 2.05 times higher risk of developing visual field defects. 
RNFL Change Report with FoDi ${ }^{\mathrm{TM}}$, All Follow-Ups

SPECTRALIS® Tracking Laser Tomography

Patient:

Patient ID:

Diagnosis:
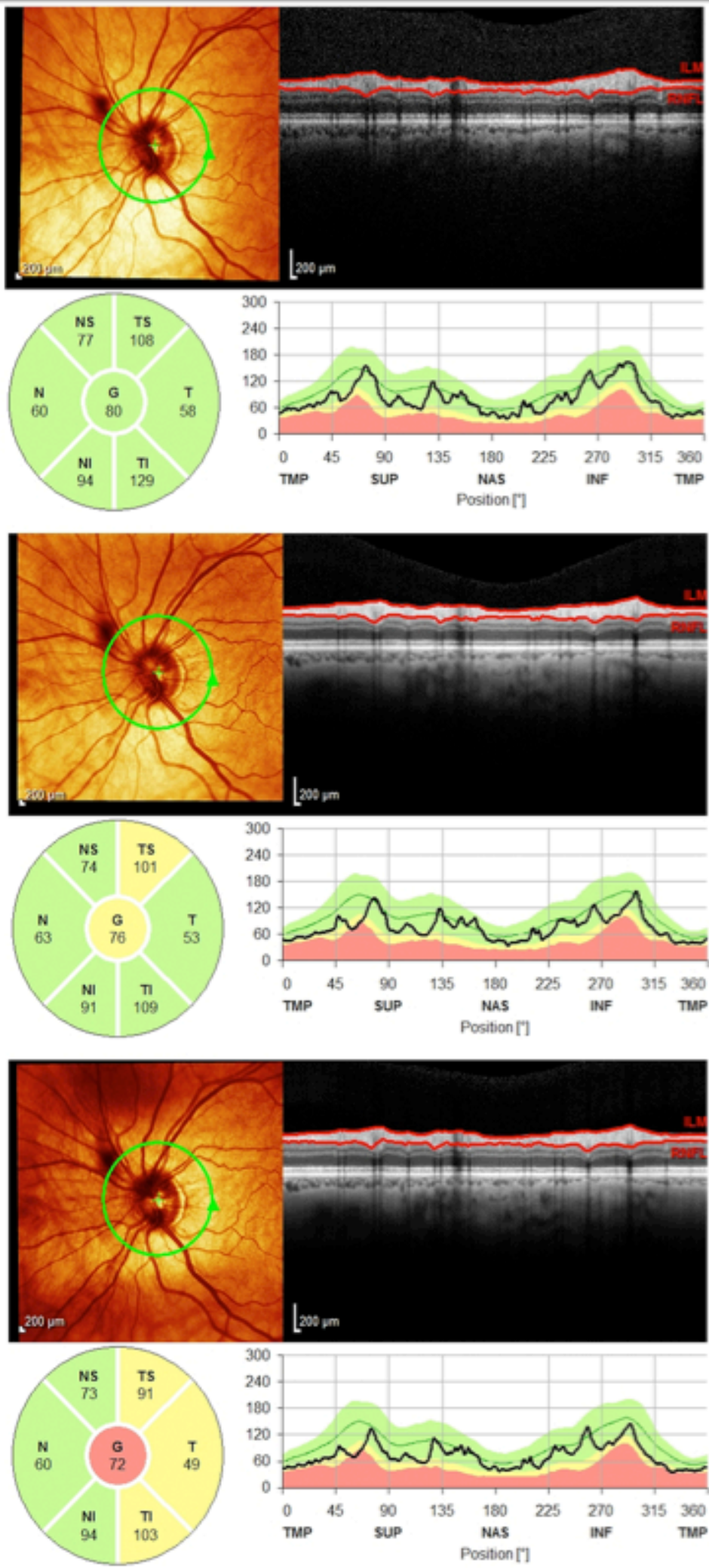

HEIDELEETG ЕПБIПЕЕГIПG

Sex:

OS
Follow-Up \#3 Apr/27/2010

IR 30" ART + OCT ART (2) Q: 25 [HR]

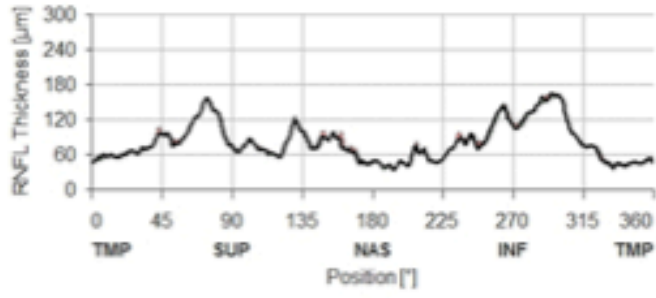

Classification

Within Normal Limits

Follow-Up \#31 May/7/2012

IR 30" ART + OCT ART [37) Q: 30 [HR]
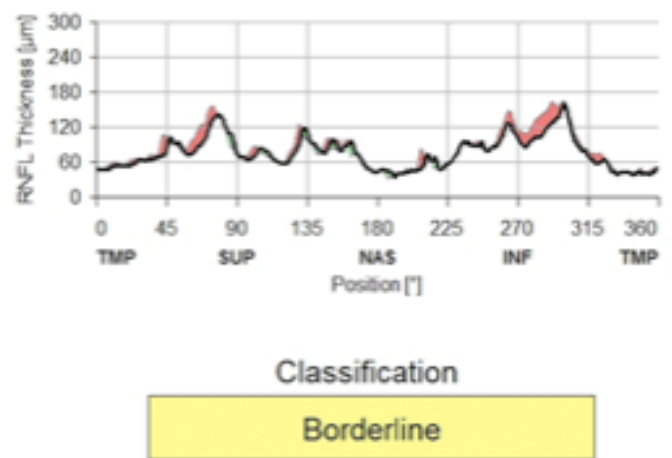

Follow-Up \#32 Nov/28/2012

IR 30' ART + OCT ART (34) Q: 33 [MR]

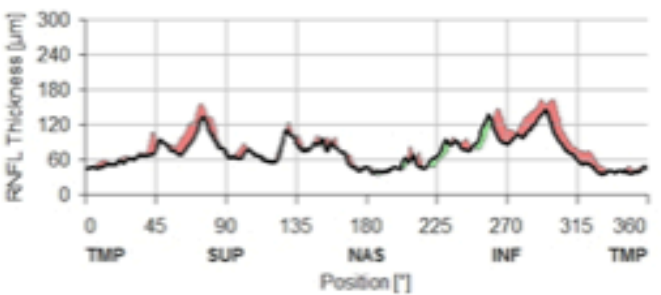

\section{Classification}

Outside Normal Limits

Fig. (1). Spectralis SD-OCT scans (Heidelberg Engineering, Heidelberg, Germany) of a glaucoma patient followed for an average of 3 years. The Change report shows progressive loss of the retinal nerve fiber layer. 


\section{CIRRUS HD-OCT}

The Cirrus HD-OCT (Carl Zeiss Meditec, Dublin, California, USA) can perform 27000 A-scans per second and capture measurements on a $6 \times 6 \mathrm{~mm}$ cube formed from 200 A-scans for each of 200 B-scans. From this cube of data, the machine automatically identifies the center of the optic disc and creates a 3.46- $\mathrm{mm}$ calculation circle around it [54].

In order to assess glaucoma progression, the Guided Progression Algorithm (GPA) was introduced in 2009 for the Cirrus HD-OCT in an attempt to facilitate detection of RNFL progression [55, 56]. It tracks RNFL changes with serial, registered RNFL thickness maps, comparing RNFL thickness of individual pixels between the baseline and follow-up images and provides a visual display of the area and location of significant change. GPA analysis provides topographical display of RNFL thickness measurements for each exam and is best to detect focal changes. The Change Map combines up to 6 exams that are automatically registered to the baseline tests for a precise point-to-point comparison. Fig. (2) shows an example of a patient with open angle glaucoma with GPA analysis of the left eye showing progressive thinning in the superotemporal and inferotemporal regions. Areas of statistically significant change are color-coded yellow when first noted and then red when the change is repeatable over consecutive tests. Average RNFL thickness values are plotted for each exam and can also be used to detect changes over time and estimate rates of change. Trends are calculated and confidence intervals are shaded when rate of change is statistically significant. Fig. (3) shows an example of a GPA analysis from the same patient with the RNFL Summary displaying numeric values for average, superior and inferior RNFL thickness parameters and rim area, average cup-todisc ratio, vertical cup-to-disc ratio and cup volume for $\mathrm{ONH}$ topographic assessment. The values are compared with the baseline exam and statistically significant change can be detected.

Several studies have supported the use of the GPA for progression detection in glaucoma [55-57]. Lee at al examined 226 eyes of 130 glaucoma patients for 2.5 years and compared the outcomes of GPA versus ONH/RNFL photographic assessment in detecting progression [58]. The study suggested that Cirrus SD-OCT GPA is a good adjunct device for those eyes with diffuse RNFL defect or an unidentifiable RNFL status in which photographic assessment is not reliable.

Leung et al. used the RNFL thickness map provided by GPA for detecting and identifying patterns of RNFL progression [59]. The most common pattern of RNFL progression was widening of RNFL defects $(85.7 \%)$, followed by development of new defects (17.9\%) and deepening of RNFL defects (7.1\%). The inferotemporal meridian was the most common location where RNFL change was detected. According to the authors, by evaluating changes in the RNFL thickness maps over time, it may be possible to better assess the pattern of RNFL progression independent of the degree of disease severity.

In another study, $\mathrm{Na}$ et al. included 279 eyes from 162 glaucoma patients followed for an average of 2.2 years, with the objective of evaluating rates of change in RNFL, macular and $\mathrm{ONH}$ parameters [18]. Eyes were classified as progressors and nonprogressors according to optic disc and RNFL photographs as well as visual field assessment. The results showed that RNFL thickness measurements from the inferior quadrant, 6 and 7-o'clock sectors decreased faster in progressors than in nonprogressors. The ONH rim area also decreased faster, whereas average and vertical cup-to-disc ratio increased faster in progressors than in nonprogressors. Macular cube volume and the thickness of temporal outer and inferior inner macular sectors also showed differences between the groups.

Recent studies have also focused on the segmentation of the different retinal layers from the macular area that can be obtained with the Cirrus HD-OCT ganglion cell analysis (GCA) [60, 61]. A recent publication reported promising results with the use of the GCA for detecting progression, although the study had a short average follow-up time of only approximately 2 years, which may limit the ability of comparing the different methods for detecting change [62].

\section{RTvue SD-OCT}

RTVue-100 (Optovue Inc., Fremont, California, USA) is a SD-OCT imaging that uses an $840 \pm 10 \mathrm{~nm}$ wavelength illumination source capable of $26000 \mathrm{~A}$-scans/s with a depth resolution of $5 \mu \mathrm{m}$ [63]. A peripapillary RNFL thickness map is generated from 13 circular scans with diameters of 1.3-4.9 $\mathrm{mm}$ and measurements are generated from the 3.45 $\mathrm{mm}$ diameter circle, centered in the optic disc. RNFL thickness is measured as the distance between the internal limiting membrane and the outer edge of the RNFL. The software uses RNFL change analysis and ganglion cell complex (GCC) progression analysis to assess rates of change [64]. A topographic GCC map is generated from one horizontal line scan $7 \mathrm{~mm}$ in length (467 A scans) followed by 15 vertical line scans $7 \mathrm{~mm}$ in length (each $400 \mathrm{~A}$ scans) and at a $0.5 \mathrm{~mm}$ interval centered $1 \mathrm{~mm}$ temporal to the fovea. GCC thickness is determined as the combined thickness of the RNFL, ganglion cell layer and inner plexiform layer. The pattern-based parameters comprise focal loss volume (FLV) and global loss volume (GLV). FLV is the total sum of statistically significant GCC volume loss divided by the GCC map area, in percentage. GLV is the sum of negative fractional deviation in the entire measurement area, in percentage. The intra-visit repeatability of the macular GCC scan by the RTVue has been shown to be good in both glaucomatous and healthy eyes [22]. For the average and quadrant RNFL parameters and the GCC parameters, intra-session ICC varied between 0.93 and 0.99 , intrasession $\mathrm{CV}$ between $1.95 \%$ and $5.69 \%$, and intra-test variability varied between 3.11 and $9.13 \mu \mathrm{m}$ [30].

Naghizadeh et al. compared the variability of RNFL measurements between scanning laser polarimetry (GDx) and RTvue in 110 eyes of 110 patients (healthy, ocular hypertensive, preperimetric and perimetric glaucoma) prospectively at 6-month intervals for 1.5 to 3 years [35]. Relative variance of all RNFL parameters measured with RTVue SD-OCT was significantly $(\mathrm{P}<0.001)$ smaller than that with the GDx, which suggests that long-term RNFL thickness measurements are less variable with the RTVue SD-OCT than with GDx. 
(A)

Name:

BaseLine 1 Current

ID:

Exam Date:

$8 / 19 / 2009$

$5 / 8 / 2013$

CZMI

DOB:

Exam Time:

9:00 AM

10:51 AM

Gender: Female

Serial Number:

4000-1063

4000-1063

Technician: Operator, Cirrus

Signal Strength: $8 / 10$

$8 / 10$

\section{Guided Progression Analysis: (GPA ${ }^{\mathrm{TM}}$ )}

\section{OD $\bigcirc \bigcirc$ OS}

$$
\begin{array}{r}
\text { 8/19/2009 9:00:45 AM } \\
4000-1063 \\
\text { SS: } 8 / 10
\end{array}
$$

Average Thickness: 78
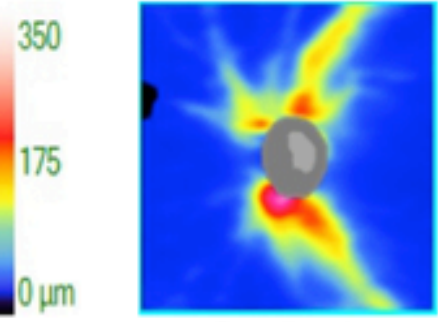

Baseline 1

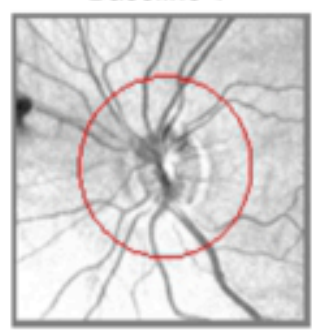

Im

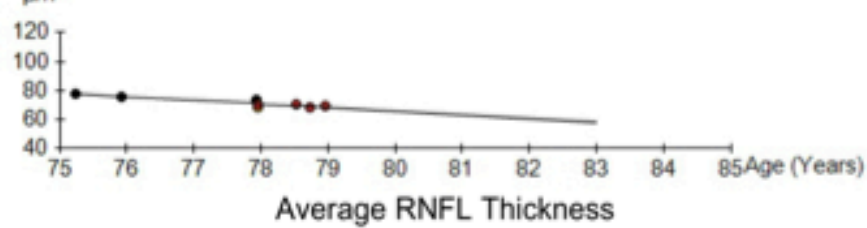

Rate of change: $-2.49+/-1.22 \mu \mathrm{m} /$ Year

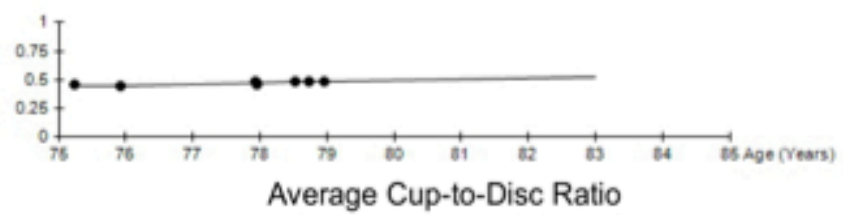

Rate of change: $0.01+/-0.01 /$ Year

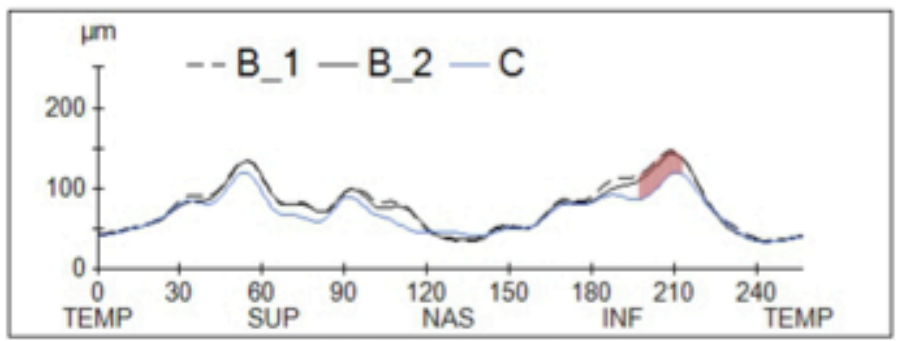

RNFL Thickness Profiles

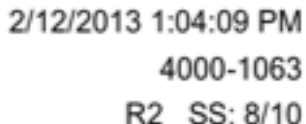

R2 SS: $8 / 10$

Average Thickness: 68
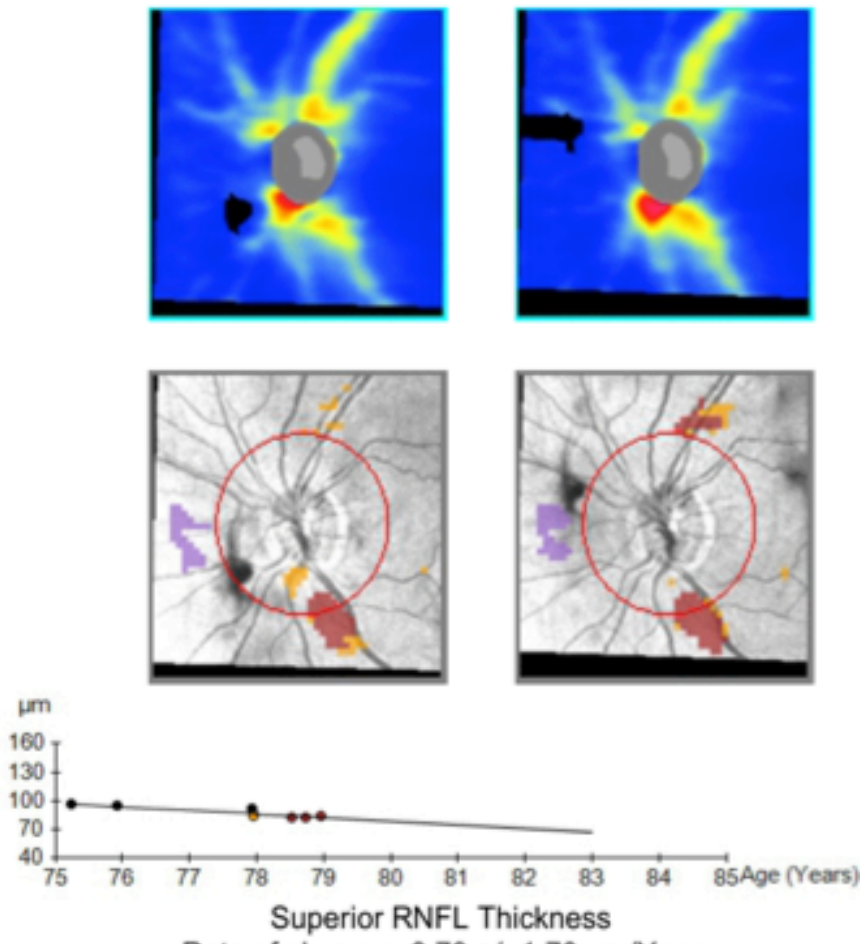

Rate of change: $-3.79+/=1.70 \mu \mathrm{m} /$ Year

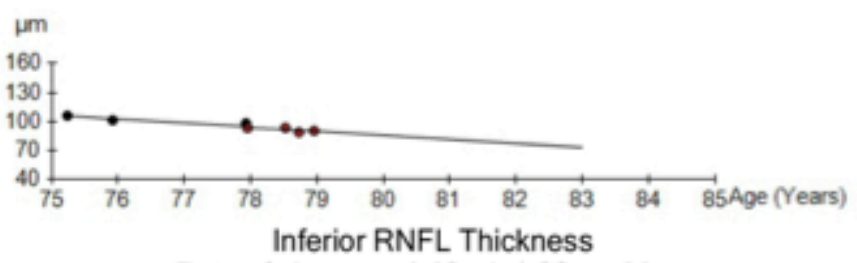

Rate of change: $-4.19+/-1.38 \mu \mathrm{m} / \mathrm{Year}$

\section{RNFL/ONH Summary OS}

RNFL Thickness Map Progression

RNFL Thickness Profiles Progression

Average RNFL Thickness Progression

Average Cup-to-Disc Progression Possible loss

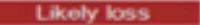

Possibse Inerense 
(B)

\section{\begin{tabular}{ll|l}
\hline Guided Progression Analysis: $\left(\mathrm{GPA}^{\mathrm{TM}}\right)$ & OD $\bigcirc$ & O OS \\
\hline
\end{tabular}}

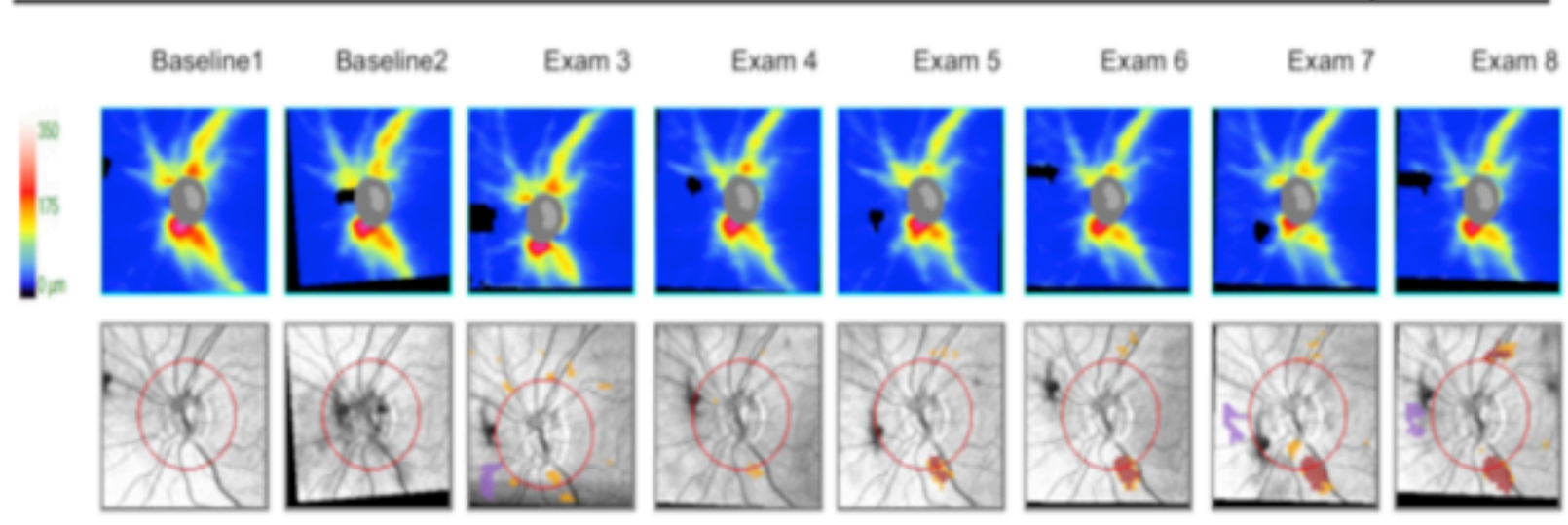

RNFL and ONH Summary Parameters

\begin{tabular}{|c|c|c|c|c|c|c|c|c|c|c|c|}
\hline & $\begin{array}{c}\text { Exam } \\
\text { Date/Time }\end{array}$ & \begin{tabular}{|l|} 
Serial \\
Number
\end{tabular} & $\begin{array}{l}\text { Registration } \\
\text { Method }\end{array}$ & sS & \begin{tabular}{|c|} 
Avg \\
RNFL \\
Thickness \\
$(\mu \mathrm{m})$
\end{tabular} & \begin{tabular}{|c|} 
Inf \\
Quadrant \\
RNFL \\
$(\mu \mathrm{m})$ \\
\end{tabular} & \begin{tabular}{|c|} 
Sup \\
Quadrant \\
RNFL \\
$(\mu \mathrm{m})$ \\
\end{tabular} & $\begin{array}{c}\text { Rim } \\
\text { Arez } \\
\left(\mathrm{mm}^{2}\right)\end{array}$ & \begin{tabular}{|c|} 
Average \\
Cup-to- \\
Disc \\
Ratio \\
\end{tabular} & \begin{tabular}{|c|} 
Vertical \\
Cup-to- \\
Disc \\
Ratio \\
\end{tabular} & $\begin{array}{l}\text { Cup } \\
\text { Volume } \\
\text { (mm') }\end{array}$ \\
\hline aseline1: & 1 c/19/2009 & $\begin{array}{l}4000- \\
1063\end{array}$ & & $8 / 10$ & 78 & 106 & 96 & 1.31 & 0.46 & 0.47 & 0.066 \\
\hline \multirow[t]{6}{*}{ Baseline2: } & \begin{tabular}{|l|c|}
2 & $4 / 27 / 2010$ \\
& $1: 44: 29$ PM \\
\end{tabular} & \begin{tabular}{|c|}
$4000-$ \\
1063 \\
\end{tabular} & R2 & $6 / 10$ & 76 & 102 & 95 & 1.34 & 0.44 & 0.46 & 0.062 \\
\hline & $3 \begin{array}{c}4 / 24 / 2012 \\
2: 24: 46 \text { PM }\end{array}$ & $\begin{array}{l}4000- \\
1063\end{array}$ & R1 & $8 / 10$ & 74 & 99 & 92 & 1.30 & 0.49 & 0.56 & 0.080 \\
\hline & \begin{tabular}{c|c|}
$5 / 2 / 2012$ \\
$3: 49: 12$ PM
\end{tabular} & \begin{tabular}{|c|}
$4000-$ \\
1063 \\
\end{tabular} & R2 & $7 / 10$ & 68 & 94 & 86 & 1.27 & 0.47 & 0.47 & 0.069 \\
\hline & $5 \begin{array}{c}5 / 7 / 2012 \\
2: 32: 00 \text { PM }\end{array}$ & \begin{tabular}{|c|}
$4000-$ \\
1063 \\
\end{tabular} & R2 & $8 / 10$ & 70 & 94 & 85 & 1.26 & 0.46 & 0.48 & 0.067 \\
\hline & $6 \begin{array}{l}11 / 28 / 2012 \\
2: 11: 15 \text { PM }\end{array}$ & \begin{tabular}{|c|}
$4000-$ \\
1063 \\
\end{tabular} & R2 & $8 / 10$ & 71 & 93 & 83 & 1.22 & 0.48 & 0.50 & 0.075 \\
\hline & \begin{tabular}{|c|c|}
$2 / 12 / 2013$ \\
$1: 04: 09$ PM \\
\end{tabular} & \begin{tabular}{|c|}
$4000-$ \\
1063 \\
\end{tabular} & R2 & $8 / 10$ & 68 & 89 & 83 & 1.24 & 0.49 & 0.51 & 0.074 \\
\hline Current: & \begin{tabular}{|c|c|}
$5 / 8 / 2013$ \\
$10: 51: 29$ AM
\end{tabular} & \begin{tabular}{|l|}
$4000-$ \\
1063
\end{tabular} & R2 & $8 / 10$ & 69 & 90 & 84 & 1.27 & 0.49 & 0.52 & 0.074 \\
\hline
\end{tabular}

\begin{tabular}{|l|l|}
\hline $\begin{array}{ll}\text { Registration Methods } \\
\text { R2-Registration based on translation and rotation of OCT fundus } \\
\text { R1 - Registration based only on translation of disc center }\end{array}$ \\
\hline Likey Loss & $\begin{array}{l}\text { Compared to baseline, statistically significant loss of tissue detected. For Average RNFL. Superior RNFL, } \\
\text { Inferior RNFL, Rim Area the values have decreased. For Cup-to-Disc Ratios and Cup Volume values have } \\
\text { increased. }\end{array}$ \\
\hline Possible Loss \\
\hline $\begin{array}{l}\text { Compared to baseline, statistically significant increase detected. For Average RNFL, Superior RNFL, } \\
\text { Inferior RNFL., Rim Area values have increased. For Cup-to-Disc Ratios and Cup Vclume values have } \\
\text { decreased. }\end{array}$ \\
\hline
\end{tabular}

Fig. (2). Cirrus HD-OCT Guided Progression Analysis (Carl Zeiss Meditec, Dublin, CA, USA) A. Printout showing progressive retinal nerve fiber layer (RNFL) thinning in the superotemporal and inferotemporal regions. Trend lines and rates of change are also provided showing statistically significant slopes of change for RNFL thickness, but not for average cup-to-disk ratio. B. Optic nerve head and RNFL Summary showing worsening of superotemporal and inferotemporal RNFL regions parameters. 


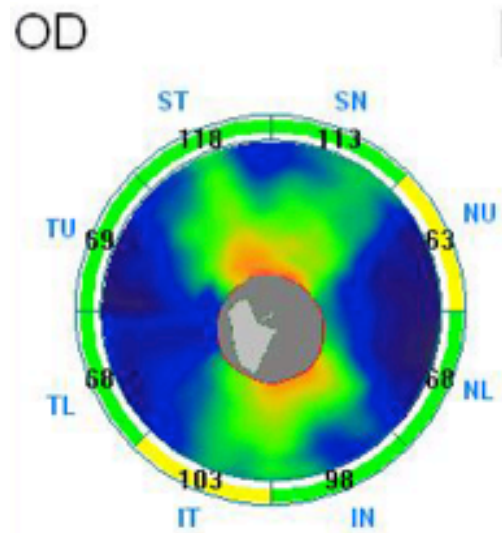

Baseline: 04/27/2009, SSI=63.3

\section{Nerve Head / RNFL Analysis}
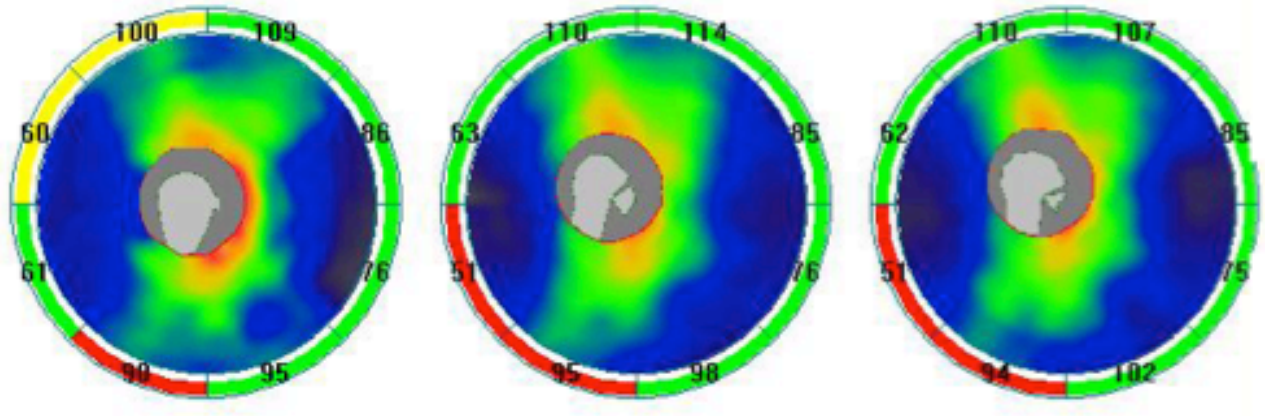

FollowUp1: 07/20/2010, SSI-63.1
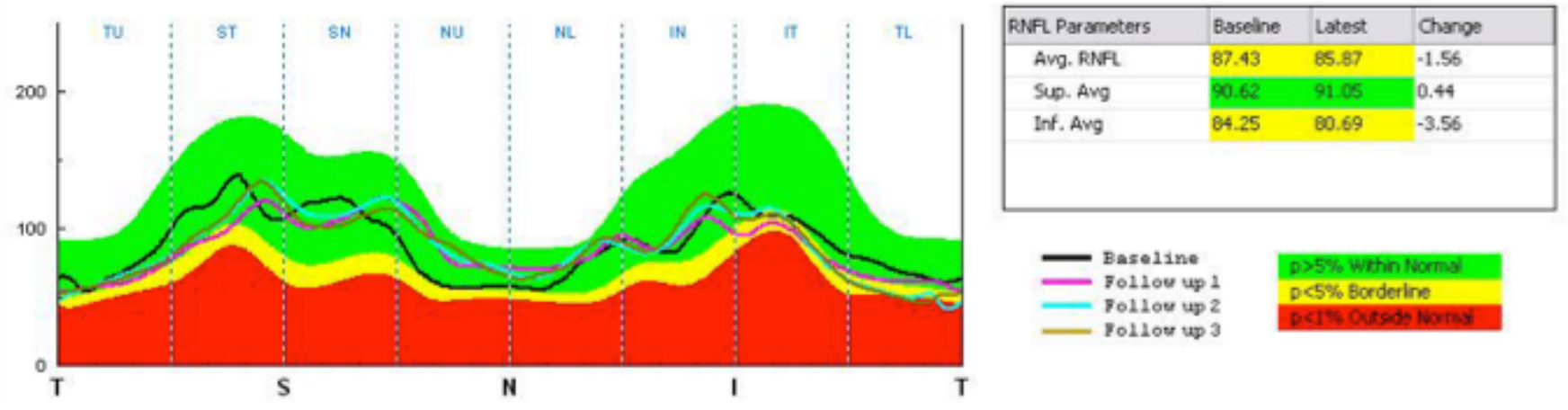

Fig. (3). Optic nerve head and retinal nerve fiber layer (ONH/RNFL) change report from RTvue from the right eye of a glaucoma patient followed for an average of 3 years showing RNFL thinning in the temporal inferior region. Average and inferior RNFL thickness decreased significantly compared to the baseline exam.

More recently the same author showed that pattern based GCC parameters of the RTVue SD-OCT may detect progression earlier than optic nerve head, RNFL thickness and average GCC [65]. In the study, 68 eyes from healthy and glaucoma patients were followed for a mean of 2 years. Progression was determined by Octopus (Haag-Streit AG, Koeniz-Berne, Switzerland) normal G2 visual field progression criteria. Comparing glaucomatous and control subjects for progression rates, no ONH, RNFL, or average GCC parameter differed significantly between the groups. In contrast, both FLV and GLV showed significantly greater progression rates $(\mathrm{P}=0.004$ and $\mathrm{P}=0.001$, respectively) for the glaucoma group $(1.460 \%$ and $3.810 \%$ per year, respectively) compared to the control eyes $(0.006 \%$ and $0.048 \%$ per year). The study included glaucoma patients with an average defect on Octopus of $+10.20 \pm 6.70$, indicating moderate and severe damage. Therefore, future studies should also attempt to evaluate these findings in eyes with earlier stages of damage.

Fig. (3) shows the printout of the ONH/RNFL change report with RTvue SD-OCT, in which up to 4 scans can be combined in a single analysis. Measurements from 8 sectors are compared to the database. On the bottom right, RNFL parameters (average, superior and inferior) from baseline to the last visit are displayed and the difference from those measurements are compared to the database.

\section{SWEPT SOURCE OCT}

Swept-source OCT (SS-OCT) is another approach for OCT imaging with speed and sensitivity characteristics similar to SD-OCT [66]. Both methods measure interference as a function of optical frequency or wavelength. However, SS-OCT uses a frequency swept laser that enables measurement of interference at different optical frequencies or wavelengths sequentially over time [67]. DRI-OCT-1 (Topcon Corp, Japan) can perform scans with speed of $100,000 \mathrm{~A}$-scans $/ \mathrm{sec}$ and in-depth resolution of $8 \mu \mathrm{m}$. SSOCT utilizes a wavelength of $1,050 \mathrm{~nm}$ allowing better visualization of choroid and sclera when compared to SDOCT with wavelength in the $850 \mathrm{~nm}$ range. Currently, no software for progression is available for this device. In a recent study, Yoshikawa et al. investigated changes in deep ONH structure following glaucoma surgery [68]. Change in lamina cribrosa depth and pre-laminar tissue thickness was evident after surgery and percent change in lamina cribrosa depth measured by SS-OCT was significantly correlated with both percent intraocular pressure change and visual field defect severity. Mansouri et al. examined 54 eyes of 27 healthy subjects using an automated segmentation algorithm of choroidal borders applied to different image acquisition protocols [69]. Signal loss because of blinking occurred in $7 \%$ of optic disc scans. After excluding artifacts, 3D optic disc scans showed an ICC of 0.99 and 0.83 for choroidal and 
retinal thickness, respectively. The results suggested that automated measurements of retinal and choroidal thickness using SS-OCT are highly repeatable. However, artifacts such as those produced by blinking may have a significant impact on the repeatability of measurements. Longitudinal studies still needs to be performed to evaluate the ability of SS-OCT to detect glaucoma progression.

\section{THE EFFECT OF AGING ON THE ASSESSMENT OF CHANGE OVER TIME}

A challenge in the accurate diagnosis of glaucoma progression includes the difficulty in discriminating true disease-related from age-related changes in structural measurements. In a prospective longitudinal study, Leung et al. examined 70 eyes of 35 healthy subjects with a mean follow-up of 30 months and showed mean rates of change of $-0.52,-1.35$ and $-1.25 \mu \mathrm{m} /$ year for average, superior, and inferior RNFL thicknesses respectively [70]. The same author investigated 222 eyes (90 patients with glaucoma and 40 normal individuals) for a mean of 3.8 years in another prospective longitudinal study [71]. Using Cirrus SD-OCT with GCA, they measured combined ganglion cell and inner plexiform layer (GCIPL) thickness, inner retina (IR) thickness, outer retina (OR) thickness and total macular thickness. Using trend analysis, $50 \%$ of eyes progressed by the GCIPL thickness, 50\% progressed by the IR thickness, $30 \%$ progressed by the total macular thickness, $27 \%$ progressed by the RNFL thickness and $10 \%$ progressed by the OR thickness. After accounting for age-related change, the proportions decreased to $14.7 \%, 20.0 \%, 16.0 \%, 26.7 \%$, and $1.3 \%$, respectively. These results suggest that age-related structural changes seem to exert significant impact on the assessment of progression by OCT.

\section{COMBINED STRUCTURE AND FUNCTION ANALYSIS}

Several approaches have been proposed to combine results from different structural and functional tests to improve detection of glaucoma progression. Medeiros et al. used Bayesian statistics to develop a joint model integrating longitudinal information obtained from structural and functional evaluations, allowing information derived from one test to influence the inferences obtained from the other test [72]. In the study, SAP and optic disc topographic measurements were obtained over time in 242 eyes of 179 glaucoma patients followed for an average of 6.4 years. The aim of the study was to evaluate whether the estimation of slopes of change could be improved by integrating structure and function. The authors found that the Bayesian slopes of change performed significantly better than conventional approaches in predicting future structural and functional measures.

More recently, Medeiros and colleagues developed an approach to estimate RGC counts from structural and functional measurements, providing a single combined metric of structure and function. The method combined estimates of RGC counts obtained from OCT RNFL thickness with those obtained from SAP [73-75]. The method has been shown to perform significantly better than conventional approaches for diagnosis, staging and detection of progression. In a longitudinal study, Medeiros and colleagues used estimates of RGC counts obtained from SAP and OCT in 213 eyes of 213 glaucoma patients followed for an average of 4.5 years. In the study, SAP was able to detect progression in only $18(8.5 \%)$ of the 213 eyes, whereas the OCT detected progression in 31 eyes $(14.6 \%)$. On the other hand, from the 213 eyes, $47(22.1 \%)$ showed statistically significant rates of RGC loss that were faster than the ageexpected decline. Therefore the index estimating the rate of RGC loss combining structure and function performed better than isolated structural and functional measures for detecting progressive glaucomatous damage [76].

\section{CONCLUSION}

SD-OCT technology provides reproducible measurements of different retinal and optic nerve structures, which have been shown to be able to detect glaucoma progression and measure rates of change. Significant improvements in detection of glaucoma progression and estimation of rates of change can be seen when structural measurements with SD-OCT are combined to those obtained from functional assessment.

\section{CONFLICT OF INTEREST}

FAM: Research support from Carl-Zeiss Meditec, Heidelberg Engineering and Topcon, Inc. Consultant to CarlZeiss Meditec, Inc. RYA: None and CPBG: None

\section{ACKNOWLEDGEMENTS}

Supported in part by National Institutes of Health/National Eye Institute grants EY021818 (F.A.M.) and core grant P30EY022589; an unrestricted grant from Research to Prevent Blindness (RPB); Brazilian National Research Council-CAPES grant 12309-13-3 (CPBG).

\section{REFERENCES}

[1] Kingman S. Glaucoma is second leading cause of blindness globally. Bull World Health Organ 2004; 82: 887-8.

[2] Weinreb RN, Aung T, Medeiros FA. The pathophysiology and treatment of glaucoma: a review. JAMA 2014; 311: 1901-11.

[3] Weinreb RN, Garway-Heath DF, Leung C, Crowston JG, Medeiros FA. World glaucoma association consensus meeting: progression of glaucoma. France: Kugler Publications Paris 2011.

[4] Alencar LM, Medeiros FA. The role of standard automated perimetry and newer functional methods for glaucoma diagnosis and follow-up. Indian J Ophthalmol 2011; 59: Suppl S53-8.

[5] Kass MA, Heuer DK, Higginbotham EJ, et al. The Ocular Hypertension Treatment Study: a randomized trial determines that topical ocular hypotensive medication delays or prevents the onset of primary open-angle glaucoma. Arch Ophthalmol 2002; 120: 701-13; discussion 829-30.

[6] Miglior S, Zeyen T, Pfeiffer N, et al. Results of the European Glaucoma Prevention Study. Ophthalmology 2005; 112: 366-75.

[7] Hood DC, Kardon RH. A framework for comparing structural and functional measures of glaucomatous damage. Prog Retin Eye Res 2007; 26: 688-710

[8] Medeiros FA, Alencar LM, Zangwill LM, Sample PA, Weinreb $\mathrm{RN}$. The relationship between intraocular pressure and progressive retinal nerve fiber layer loss in glaucoma. Ophthalmology 2009; 116: 1125-33.e1-3

[9] Medeiros FA, Alencar LM, Zangwill LM, et al. Prediction of functional loss in glaucoma from progressive optic disc damage. Arch Ophthalmol 2009; 127: 1250-6. 
[10] Vermeer KA, Lemij HG. Challenges in estimating the accuracy of imaging-based detection methods for glaucomatous progression. $\mathrm{Br}$ J Ophthalmol 2013; 97: 385-6.

[11] Azuara-Blanco A, Katz LJ, Spaeth GL, et al. Clinical agreement among glaucoma experts in the detection of glaucomatous changes of the optic disk using simultaneous stereoscopic photographs. Am J Ophthalmol 2003; 136: 949-50.

[12] Parrish RK, Schiffman JC, Feuer WJ, et al. Test-retest reproducibility of optic disk deterioration detected from stereophotographs by masked graders. Am J Ophthalmol 2005; 140: 762-4.

[13] Jampel HD, Friedman D, Quigley H, et al. Agreement among glaucoma specialists in assessing progressive disc changes from photographs in open-angle glaucoma patients. Am J Ophthalmol 2009; 147: 39-44.e1.

[14] Medeiros FA, Zangwill LM, Alencar LM, Sample PA, Weinreb RN. Rates of progressive retinal nerve fiber layer loss in glaucoma measured by scanning laser polarimetry. Am J Ophthalmol 2010; 149: $908-15$

[15] Lisboa R, Chun YS, Zangwill LM, et al. Association between rates of binocular visual field loss and vision-related quality of life in patients with glaucoma. JAMA Ophthalmol 2013; 131: 486-94.

[16] Medeiros FA, Zangwill LM, Alencar LM, et al. Detection of glaucoma progression with stratus OCT retinal nerve fiber layer, optic nerve head, and macular thickness measurements. Invest Ophthalmol Vis Sci 2009; 50: 5741-8.

[17] Grewal DS, Tanna AP. Diagnosis of glaucoma and detection of glaucoma progression using spectral domain optical coherence tomography. Curr Opin Ophthalmol 2013; 24: 150-61.

[18] Na JH, Sung KR, Lee JR, et al. Detection of glaucomatous progression by spectral-domain optical coherence tomography. Ophthalmology 2013; 120: 1388-95.

[19] Wessel JM, Horn FK, Tornow RP, et al. Longitudinal analysis of progression in glaucoma using spectral-domain optical coherence tomography. Invest Ophthalmol Vis Sci 2013; 54: 3613-20.

[20] Park HY, Shin HY, Park CK. Imaging the posterior segment of the eye using swept-source optical coherence tomography in myopic glaucoma eyes: comparison with enhanced-depth imaging. Am J Ophthalmol 2014; 157: 550-7.

[21] Park SB, Sung KR, Kang SY, Kim KR, Kook MS. Comparison of glaucoma diagnostic Capabilities of Cirrus HD and Stratus optical coherence tomography. Arch Ophthalmol 2009; 127: 1603-9.

[22] Tan O, Chopra V, Lu AT, et al. Detection of macular ganglion cell loss in glaucoma by Fourier-domain optical coherence tomography. Ophthalmology 2009; 116: 2305-14.e1-2.

[23] Cremasco F, Massa G, Vidotti GV, Pedroso de Carvalho Lupinacci Á, Costa VP. Intrasession, intersession, and interexaminer variabilities of retinal nerve fiber layer measurements with spectraldomain OCT. Eur J Ophthalmol 2011; 21: 264-70.

[24] Pierro L, Gagliardi M, Iuliano L, Ambrosi A, Bandello F. Retinal nerve fiber layer thickness reproducibility using seven different OCT instruments. Invest Ophthalmol Vis Sci 2012; 53: 5912-20.

[25] Araie M. Test-retest variability in structural parameters measured with glaucoma imaging devices. Jpn J Ophthalmol 2013; 57: 1-24.

[26] Fleiss JL. The design and analysis of clinical experiments. NJ: Wiley Hoboken 2011

[27] Fleiss JL, Levin B, Paik MC. Statistical methods for rates and proportions. NJ: Wiley Hoboken 2003.

[28] Pierro L, Giatsidis SM, Mantovani E, Gagliardi M. Macular thickness interoperator and intraoperator reproducibility in healthy eyes using 7 optical coherence tomography instruments. Am J Ophthalmol 2010; 150: 199-204.e1.

[29] Mwanza JC, Chang RT, Budenz DL, et al. Reproducibility of peripapillary retinal nerve fiber layer thickness and optic nerve head parameters measured with cirrus HD-OCT in glaucomatous eyes. Invest Ophthalmol Vis Sci 2010; 51: 5724-30.

[30] Garas A, Vargha P, Holló G. Reproducibility of retinal nerve fiber layer and macular thickness measurement with the RTVue-100 optical coherence tomograph. Ophthalmology 2010; 117: 738-46.

[31] Sung KR, Sun JH, Na JH, Lee JY, Lee Y. Progression detection capability of macular thickness in advanced glaucomatous eyes. Ophthalmology 2012; 119: 308-13.

[32] Töteberg-Harms M, Sturm V, Knecht PB, Funk J, Menke MN. Repeatability of nerve fiber layer thickness measurements in patients with glaucoma and without glaucoma using spectral- domain and time-domain OCT Graefes. Arch Clin Exp Ophthalmol 2012; 250: 279-87.

[33] Ghasia FF, El-Dairi M, Freedman SF, Rajani A, Asrani S. Reproducibility of spectral-domain optical coherence tomography measurements in adult and pediatric glaucoma. J Glaucoma 2013 [Epub ahead of print].

[34] Medeiros FA, Doshi R, Zangwill LM, Vasile C, Weinreb RN. Long-term variability of GDx VCC retinal nerve fiber layer thickness measurements. J Glaucoma 2007; 16: 277-81.

[35] Naghizadeh F, Garas A, Vargha P, Holló G. Comparison of longterm variability of retinal nerve fiber layer measurements made with the RTVue OCT and scanning laser polarimetry. Eur J Ophthalmol 2012 [Epub ahead of print].

[36] Huang D, Swanson EA, Lin CP, et al. Optical coherence tomography. Science 1991; 254: 1178-81.

[37] Hee MR, Izatt JA, Swanson EA, et al. Optical coherence tomography of the human retina. Arch Ophthalmol 1995; 113: 32532.

[38] Chen TC, Cense B, Pierce MC, et al. Spectral domain optical coherence tomography: ultra-high speed, ultra-high resolution ophthalmic imaging. Arch Ophthalmol 2005; 123: 1715-20.

[39] van Velthoven ME, Faber DJ, Verbraak FD, van Leeuwen TG, de Smet MD. Recent developments in optical coherence tomography for imaging the retina. Prog Retin Eye Res 2007; 26: 57-77.

[40] Menke MN, Dabov S, Knecht P, Sturm V. Reproducibility of retinal thickness measurements in healthy subjects using spectralis optical coherence tomography. Am J Ophthalmol 2009; 147: 46772.

[41] Vizzeri G, Weinreb RN, Gonzalez-Garcia AO, et al. Agreement between spectral-domain and time-domain OCT for measuring RNFL thickness. Br J Ophthalmol 2009; 93: 775-81.

[42] Knight OJ, Chang RT, Feuer WJ, Budenz DL. Comparison of retinal nerve fiber layer measurements using time domain and spectral domain optical coherent tomography. Ophthalmology 2009; 116: 1271-7.

[43] Sehi M, Grewal DS, Sheets CW, Greenfield DS. Diagnostic ability of Fourier-domain vs time-domain optical coherence tomography for glaucoma detection. Am J Ophthalmol 2009; 148: 597-605.

[44] Wollstein G, Schuman JS, Price LL, et al. Optical coherence tomography longitudinal evaluation of retinal nerve fiber layer thickness in glaucoma. Arch Ophthalmol 2005; 123: 464-70.

[45] Leung CK. Diagnosing glaucoma progression with optical coherence tomography. Curr Opin Ophthalmol 2014; 25: 104-11.

[46] Vizzeri G, Bowd C, Medeiros FA, Weinreb RN, Zangwill LM. Effect of improper scan alignment on retinal nerve fiber layer thickness measurements using Stratus optical coherence tomograph. J Glaucoma 2008; 17: 341-9.

[47] Cheung CY, Leung CK, Lin D, Pang CP, Lam DS. Relationship between retinal nerve fiber layer measurement and signal strength in optical coherence tomography. Ophthalmology 2008; 115: 134751, 51.e1-2.

[48] Wojtkowski M, Srinivasan V, Fujimoto JG, et al. Threedimensional retinal imaging with high-speed ultrahigh-resolution optical coherence tomography. Ophthalmology 2005; 112: 173446.

[49] González-García AO, Vizzeri G, Bowd C, et al. Reproducibility of RTVue retinal nerve fiber layer thickness and optic disc measurements and agreement with Stratus optical coherence tomography measurements. Am J Ophthalmol 2009; 147: 1067-74, 74.e1

[50] Lee KH, Kang MG, Lim H, Kim CY, Kim NR. A formula to predict spectral domain optical coherence tomography (OCT) retinal nerve fiber layer measurements based on time domain OCT measurements. Korean J Ophthalmol 2012; 26: 369-77.

[51] Bussel II, Wollstein G, Schuman JS. OCT for glaucoma diagnosis, screening and detection of glaucoma progression. Br J Ophthalmol 2013 [Epub ahead of print].

[52] Leite MT, Rao HL, Zangwill LM, Weinreb RN, Medeiros FA. Comparison of the diagnostic accuracies of the Spectralis, Cirrus, and RTVue optical coherence tomography devices in glaucoma. Ophthalmology 2011; 118: 1334-9.

[53] Miki A, Medeiros FA, Weinreb RN, et al. Rates of retinal nerve fiber layer thinning in glaucoma suspect eyes. Ophthalmology 2014; 121: 1350-8.

[54] Sung KR, Kim DY, Park SB, Kook MS. Comparison of retinal nerve fiber layer thickness measured by Cirrus HD and Stratus 
optical coherence tomography. Ophthalmology 2009; 116: 126470, 70.e1

[55] Na JH, Sung KR, Baek S, Lee JY, Kim S. Progression of retinal nerve fiber layer thinning in glaucoma assessed by cirrus optical coherence tomography-guided progression analysis. Curr Eye Res 2013; 38: 386-95.

[56] Leung CK, Cheung CY, Weinreb RN, et al. Evaluation of retinal nerve fiber layer progression in glaucoma: a study on optical coherence tomography guided progression analysis. Invest Ophthalmol Vis Sci 2010; 51: 217-22.

[57] Tenkumo K, Hirooka K, Baba T, et al. Evaluation of relationship between retinal nerve fiber layer thickness progression and visual field progression in patients with glaucoma. Jpn J Ophthalmol 2013; 57: 451-6.

[58] Lee JR, Sung KR, Na JH, Shon K, Lee KS. Discrepancy between optic disc and nerve fiber layer assessment and optical coherence tomography in detecting glaucomatous progression. Jpn J Ophthalmol 2013; 57: 546-52.

[59] Leung CK, Yu M, Weinreb RN, et al. Retinal nerve fiber layer imaging with spectral-domain optical coherence tomography: patterns of retinal nerve fiber layer progression. Ophthalmology 2012; 119: 1858-66

[60] Mwanza JC, Oakley JD, Budenz DL, et al. Macular ganglion cellinner plexiform layer: automated detection and thickness reproducibility with spectral domain-optical coherence tomography in glaucoma. Invest Ophthalmol Vis Sci 2011; 52: 8323-9.

[61] Francoz M, Fenolland JR, Giraud JM, et al. Reproducibility of macular ganglion cell-inner plexiform layer thickness measurement with cirrus HD-OCT in normal, hypertensive and glaucomatous eyes. Br J Ophthalmol 2014; 98: 322-8.

[62] Na JH, Sung KR, Baek S, et al. Detection of glaucoma progression by assessment of segmented macular thickness data obtained using spectral domain optical coherence tomography. Invest Ophthalmol Vis Sci 2012; 53: 3817-26.

[63] Rao HL, Zangwill LM, Weinreb RN, et al. Comparison of different spectral domain optical coherence tomography scanning areas for glaucoma diagnosis. Ophthalmology 2010; 117: 1692-9, 9.e1

[64] Huang JY, Pekmezci M, Mesiwala N, Kao A, Lin S. Diagnostic power of optic disc morphology, peripapillary retinal nerve fiber layer thickness, and macular inner retinal layer thickness in glaucoma diagnosis with fourier-domain optical coherence tomography. J Glaucoma 2011; 20: 87-94.
[65] Naghizadeh F, Garas A, Vargha P, Holló G. Detection of early glaucomatous progression with different parameters of the RTVue optical coherence tomograph. J Glaucoma 2014; 23: 195-8.

[66] Yasuno $\mathrm{Y}$, Hong $\mathrm{Y}$, Makita $\mathrm{S}$, et al. In vivo high-contrast imaging of deep posterior eye by 1-microm swept source optical coherence tomography and scattering optical coherence angiography. Opt Express 2007; 15: 6121-39.

[67] Srinivasan VJ, Adler DC, Chen Y, et al. Ultrahigh-speed optical coherence tomography for three-dimensional and en face imaging of the retina and optic nerve head. Invest Ophthalmol Vis Sci 2008; 49: 5103-10.

[68] Yoshikawa M, Akagi T, Hangai M, et al. Alterations in the neural and connective tissue components of glaucomatous cupping after glaucoma surgery using swept-source optical coherence tomography. Invest Ophthalmol Vis Sci 2014; 55: 477-84.

[69] Mansouri K, Medeiros FA, Tatham AJ, Marchase N, Weinreb RN. Evaluation of retinal and choroidal thickness by swept-source optical coherence tomography: repeatability and assessment of artifacts. Am J Ophthalmol 2014; 157: 1022-32.

[70] Leung CK, Yu M, Weinreb RN, et al. Retinal nerve fiber layer imaging with spectral-domain optical coherence tomography: a prospective analysis of age-related loss. Ophthalmology 2012; 119: $731-7$.

[71] Leung CK, Ye C, Weinreb RN, et al. Impact of age-related change of retinal nerve fiber layer and macular thicknesses on evaluation of glaucoma progression Ophthalmology 2013; 120: 2485-92.

[72] Medeiros FA, Zangwill LM, Girkin CA, Liebmann JM, Weinreb $\mathrm{RN}$. Combining structural and functional measurements to improve estimates of rates of glaucomatous progression. Am J Ophthalmol 2012; 153: 1197-205.e1

[73] Medeiros FA, Lisboa R, Weinreb RN, et al. Retinal ganglion cell count estimates associated with early development of visual field defects in glaucoma. Ophthalmology 2013; 120: 736-44

[74] Meira-Freitas D, Lisboa R, Tatham A, et al. Predicting progression in glaucoma suspects with longitudinal estimates of retinal ganglion cell counts. Invest Ophthalmol Vis Sci 2013; 54: 4174-83

[75] Tatham AJ, Weinreb RN, Zangwill LM, et al. Estimated retinal ganglion cell counts in glaucomatous eyes with localized retinal nerve fiber layer defects. Am J Ophthalmol 2013; 156: 578-87.e1

[76] Medeiros FA, Zangwill LM, Anderson DR, et al. Estimating the rate of retinal ganglion cell loss in glaucoma. Am J Ophthalmol 2012; 154: 814-24.e1

(C) Abe et al.; Licensee Bentham Open

This is an open access article licensed under the terms of the Creative Commons Attribution Non-Commercial License (http://creativecommons.org/licenses/by-nc/3.0/) which permits unrestricted, non-commercial use, distribution and reproduction in any medium, provided the work is properly cited. 